\title{
TTACCA: TWO-HOP BASED TRAFFIC AWARE CONGESTION CONTROL ALGORITHM FOR WIRELESS SENSOR NETWORKS
}

\author{
Prabha $\mathrm{R}^{1}$, Prashanth Kumar Gouda ${ }^{1}$, Manjula $\mathrm{S} \mathrm{H}^{1}$, K R Venugopal ${ }^{1}$ \\ and L M Patnaik ${ }^{2}$ \\ ${ }^{1}$ Department of Computer Science and Engineering, \\ University Visvesvaraya College of Engineering \\ Bangalore University, Bangalore, India \\ ${ }^{2}$ Honorary Professor, Indian Institute of Science, Bangalore 560 001, India \\ heshakil@yahoo.com, prashanth333.ecelgmail.com, \\ shmanjula@gmail.com, \\ venugopalkr@gmail.com, patnaiklm@cedt.iisc.ernet.in
}

\begin{abstract}
Congestion in Wireless Sensor Networks has negative impact on the Quality of Service. Congestion effects the performance metrics, namely throughput and per-packet energy consumption, network lifetime and packet delivery ratio. Reducing congestion allows better utilization of the network resources and thus enhances the Quality of Service metrics of the network. Traffic Aware Dynamic Routing to Alleviate Congestion in Wireless Sensor Networks reduces congestion by considering one hop neighbor routing in the network. This paper proposed an algorithm for Quality of Service Based Traffic-Aware Data forwarding for congestion control in wireless sensor networks based on two hop neighbor information. On detection of congestion, the algorithm forwards data packets around the congestion areas by spreading the excessive packets through multiple paths. The path with light load or under loaded nodes is efficiently utilized whenever congestion occurs. The main aspect of the algorithm is to build path to the destination using two independent potential fields depth and queue length. Queue length field solves the traffic-aware problem. Depth field creates a backbone to forward packets to the sink. Both fields are combined to yield a hybrid potential field to make dynamic decision for data forwarding. Network Simulator used for simulating the algorithm is NS2. The proposed algorithm performs better.
\end{abstract}

\section{KEYWORDS}

Congestion Control, Depth Potential Field, Queue Length Potential Field, Traffic-Aware, Wireless Sensor Networks.

\section{INTRODUCTION}

Wireless Sensor Networks (WSN) are emerged as an innovative technology which are applied in a wide range of areas like environment monitoring, military, medical systems etc.. The main task of any WSN is to collect and process information in a coordinated way in the region of

Natarajan Meghanathan et al. (Eds) : NeTCoM, CSIT, GRAPH-HOC, SPTM - 2014

pp. 185-194, 2014. (C) CS \& IT-CSCP 2014

DOI : $10.5121 /$ csit.2014.41315 
deployment and deliver the data to the sink node via the communication path. Quality of Service (QoS) requirements in WSN differs from wired networks. The existing researches related to the QoS in WSN are classified into three categories: (i) traditional end-to-end QoS, (ii) reliability assurance, and (iii) application-specific QoS. In case of wired networks QoS routing is performed usually through resource reservation. WSN networks characteristics such as loose network state information, dynamically varying network topology, unrestricted mobility of hosts, and limited availability of bandwidth and battery power make QoS very demanding. While performing a certain task in WSN, when the data traffic becomes heavier in sensor node, packets are stored in the buffer. When the buffer reaches to threshold level, there will be traffic at the node for the data packets. These results in increased contention, increased retransmissions, decreased packet delivery ratios and increased energy consumption. Data loss due to congestion ultimately threatens the Quality of Service parameters namely throughput, packet delivery ratio, latency and energy efficiency. Congestion in the network threatens the network performance [1].

\section{MOTIVATION}

Traffic-Aware Dynamic Routing to Alleviate Congestion in Wireless Sensor Networks (TADR) [2] work focused on alleviating the congestion in keeping view of fidelity level of the applications as the data generated during crisis state is more important. Whenever there is congestion in the network, congestion is handled by scattering the excessive packets to alternative paths with less load. The main drawbacks of TADR are more energy consumption and throughput is less due the one hop neighbour information used for congestion control.

\section{CONTRIBUTION}

The QoS parameters emphasized in this paper are throughput, delay and energy. The objective of our Two-hop based Traffic Aware Congestion Control Algorithm (TTACCA) for Wireless Sensor Network is to alleviate congestion and improve throughput by distributing packets in both time and space. Two independent potential fields Depth field and Queue length fields plays an major role in the network to control the congestion due the occurrence of an event. The proposed algorithm works based on two-hop routing technique.

\section{RELATED WORK}

This section provides a brief overview of existing congestion control techniques. Congestion control in WSN has gained high importance in the field of research. Muhammad et al., [3] proposed a Prioritized Heterogeneous Traffic-oriented Congestion Control Protocol which performs hop-by-hop rate adjustment controlling the congestion and ensures efficient rate for the prioritized diverse traffic. This protocol uses intra-queue and inter-queue priorities along with weighted fair queuing for ensuring feasible transmission rates of Farooq et al., [4] came up with an algorithm that takes a novel and different approach towards congestion control in wireless multimedia sensor networks.Upon detection of congestion, the congested node makes an estimate of the data rate that should be used by the node itself and its one hop away upstream neighbours. While estimating the data rate, the congested node considers the characteristics of the different traffic classes along with their total bandwidth usage. Sohail et al., [5] designed Congestion Control Protocol for mobile sensor networks. It uses the existing TDMA technique with combination of statistical Time Division Multiplexing and enhanced newly proposed Time Sharing Time Division Multiple Access scheme to avoid congestion in WSN. Charalambos et al., [6], proposed two methods to tackle congestion in WSNs. The two methods are either by reducing the load or by increasing resources. Hierarchical Tree Alternative Path algorithm is 
implemented for resource control. The algorithm attempts through simple steps and minor computations to mitigate congestion in wireless sensor networks by creating dynamic alternative paths to the sink.

In [7] an algorithm which is called Rapid Congestion Control Algorithm (RCCA) for data flow rate control and Cluster Head $(\mathrm{CH})$ selection is proposed. Most of the wireless sensor networks research topics consider how to save the energy of the sensor nodes. In some applications of WSN, like the monitoring of an earthquake or forest wildfire, transmitting emergency data packets to the sink node as soon as possible is much more important than saving power. Liu et al., [8] focused on Priority-based Hybrid Protocol WSN model to provide a feasible WSN architecture, which can save the energy of the sensor nodes in normal situation, and transmits emergency data packets in an efficient manner to the sink node. Gajendra et al., [9] has presented the multiple sink mechanism in which sensor nodes are able to deliver data to multiple sink in the network. Congestion control mechanism used in this work improves the packet delivery ratio, reliability, throughput of the network. It reduces the packet loss ratio, which reduces the number of retransmission, saves the energy of sensor node. This improves the network lifetime. It is able to handle the Black Hole Problem in the wireless sensor network. Kiran et al., [10] focused on many problems starting with the basic problem of eliminating the phenomenon of congestion collapse, and include the problems of effectively using the available network resources in different types of environments. Many other techniques have the ability to measure the loss rate, delay and bottleneck buffer size, and level of congestion in wireless sensor networks.

\section{NETWORK MODEL}

In this section Network Model used and the Assumptions made are discussed along with preliminaries required for the design of our proposed TTACCA Algorithm.

\subsection{Network Model and Assumptions Made}

The network is modelled as bowl structure as illustrated in Figure 1. The sink resides at the bottom, and all data packets flow down along the surface just like water. When the traffic load in the network is light, the surface of the bowl is smoother and hence our algorithm acts just like the shortest path routing. In heavily loaded cases (e.g., burst of data packets caused by detection of a monitoring event), the congestion will form bulges on the bowl surface which blocks the packets to flow directly down to the bottom along the shortest path. The excessive packets are driven by the potential field to the appropriate alternative path without obstacles, i.e., idle or less loaded nodes. When the congestion disappears, the bowl surface becomes smoother, and the packets continue to move along the shortest path. Essentially, through spreading the packet transmissions spatially and temporarily, our TTACCA scheme alleviates congestion, while improving the throughput at the same time [2].

Table 1: Symbols used in the Algorithm

\begin{tabular}{l|l}
\hline Symbols & Meaning \\
\hline$N \_I D$ & Neighbour node ID \\
\hline$U \_$msg & Updated Message \\
\hline local depth & Depth value of the parent node \\
\hline Local queue length & Queue length value of parent node \\
\hline$Q\left(N_{-} I D\right)$ & Normalized queue length of the neighbour node \\
\hline$C$ & $\begin{array}{l}\text { Cost of radio link between parent node and } \\
\text { neighbour node }\end{array}$ \\
\hline$D$ & Depth of neighbour node \\
\hline
\end{tabular}




\begin{tabular}{l|l}
\hline$Q$ & Queue length of node \\
\hline$F_{d}\left(N_{-} I D\right)$ & $\begin{array}{l}\text { Depth force between parent node and neighbour } \\
\text { node }\end{array}$ \\
\hline$F_{q}\left(N_{-} I D\right)$ & $\begin{array}{l}\text { Queue length force between parent node and } \\
\text { neighbour node }\end{array}$ \\
\hline$F_{m}\left(N_{-} I D\right)$ & $\begin{array}{l}\text { Combined force between parent node and neighbour } \\
\text { node }\end{array}$ \\
\hline$A$ & Combined Co-efficient \\
\hline$P$ & Data packet \\
\hline
\end{tabular}

\subsection{Preliminaries}

This section describes the construction of routing potential fields required for our proposed TTACCA algorithm namely the depth field and the queue length field.

(i) Depth Potential Field: Provides the basic routing function (which the smooth bowl does), namely, to make each packet flow towards the sink. TTACCA defines the depth potential field $V_{d}$ as $V_{d}=\operatorname{Depth}(v)$ where $\operatorname{Depth}(v)$ is the depth of node $v$. The depth field from node $v$ to its neighbour $w \in \operatorname{nbr}(v)$ is given by

$F_{d}(v, w)=\left(V_{d}(v)-V_{d}(w)\right) / c_{v, w}$

$F_{d}(v, w)$ denotes the force between the parent node $v$ and the neighbour node $w$. This force is calculated by taking difference of depth field from parent node $v$ and its neighbour $w$ divided by cost of radio link between the node $v$ and neighbour $w$.

(ii) Queue Length Field: The priority routing function which determines the number of packets at each and every node in the network. In the bowl structure network model, packets move from a node to a neighbour with lower potential. To avoid a hotspot which is identified by a large queue, the potential at this node should be raised. Now, we define the queue length potential field.

$V q(v)=Q(v)$

$Q(v)$ denotes the normalized queue at node $v$ and it is defined as $Q(v)=$ Number of packets in the queue/Buffer size at node $v$.

\section{PROBLEM DEFinition}

The objective of our TTACCA algorithm is to alleviate congestion and improve throughput by distributing packets in both time and space. The main focus of this work is on detection and reduction of congestion in a particular node in WSN. The algorithm works based on Two-hop routing technique. Two-hop based Traffic-Aware Congestion Control Algorithm forwards data packets around the congestion areas by spreading the excessive along multiple paths. The idle or under loaded nodes are efficiently utilized in response to congestion. 


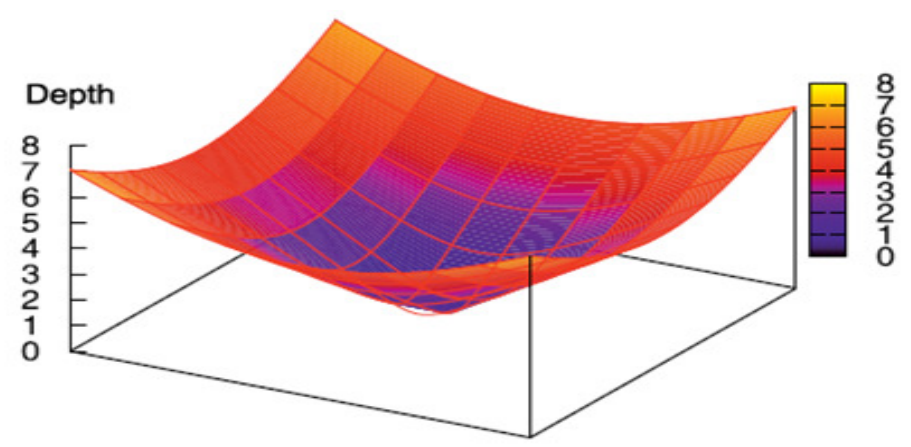

Figure 1: Bowl structure of the network

\section{ALGORITHM}

This section explains the TTACCA algorithm given in Table 2. TTACCA needs the status information from neighbour nodes, such as queue length and depth, to construct the potential fields.

The WSN is modelled as a bowl structure. In the bowl model each node will send data packets to the neighbours before, it reaches sink node. The data packets generated from source sensor nodes acts as input to the TTACCA algorithm. The neighbour nodes receiving the data packets, finds the shortest paths to the sink by choosing the depth field and congestion free path using queue length potential field. The neighbours receiving the $N \_I D, U \_m s g$ from the neighbours, updates the parent node information into the routing table. The routing table contains the information like $S I N K_{-} I D, N_{-} I D, C, d, q, Q\left(N_{-} I D\right), F_{d}\left(N_{-} I D\right)$. Routing table contains information of all one hop neighbours and two hop neighbours routing information. Which includes $N_{-} I D, C, d, q, Q\left(N_{-} I D\right), F_{d}\left(N_{-} I D\right), F q\left(N_{-} I D\right), F_{m}\left(N_{-} I D\right)$ (Combined force field between parent and its neighbour).

Table 2: Two-Hop based Traffic Aware Congestion Control Algorithm (TTACCA)

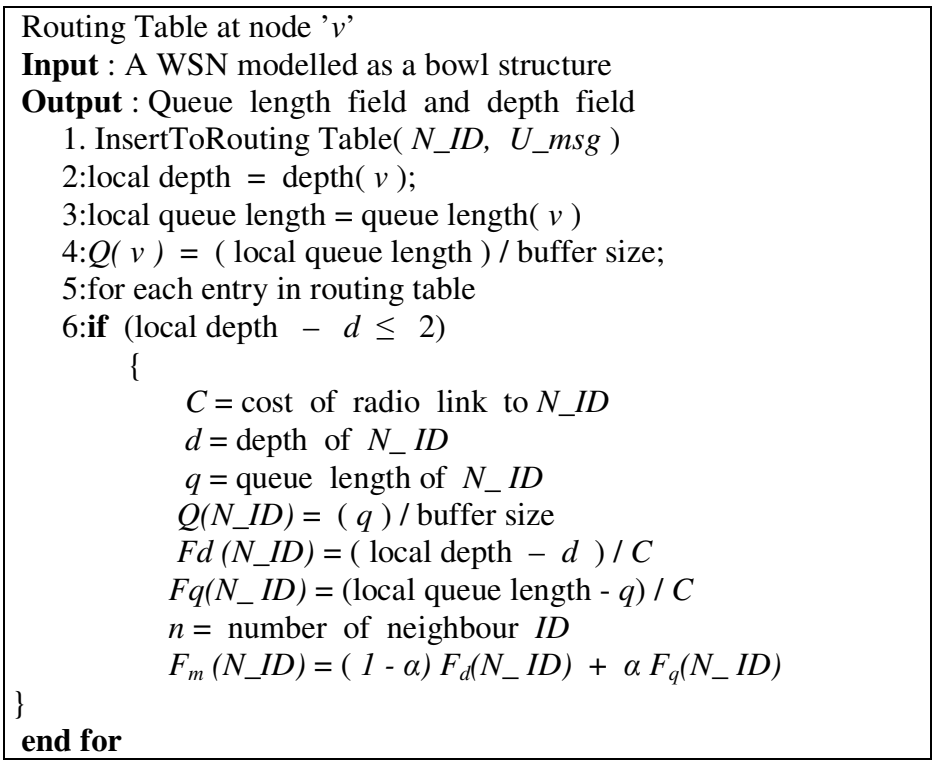




\section{Performance evaluation}

This section details the simulation parameters used for the simulation of the TTACCA algorithm and Performance analysis of the algorithm through the graphs.

\subsection{Simulation Setup}

Table 3: Simulation Parameters

\begin{tabular}{l|l}
\hline Parameters & Value \\
\hline Area Size & $500 \mathrm{~m} * 500 \mathrm{~m}$ \\
\hline Number of nodes & $18-99$ \\
\hline Deployment Type & Random \\
\hline Transmission Range & $212 \mathrm{mtrs}$ \\
\hline Sink Coordination & $250 \mathrm{~m} * 250 \mathrm{~m}$ \\
\hline Initial Energy & $1 \mathrm{~J}$ \\
\hline $\begin{array}{l}\text { Link layer } \\
\text { transmission }\end{array}$ & $8 \mathrm{kbps}$ \\
\hline $\begin{array}{l}\text { Interface Queue } \\
\text { Type }\end{array}$ & Queue/DropTail/PriQueue \\
\hline Antenna & Antenna/OmniAnteena \\
\hline 0.1 seconds & Least Utilization Time \\
\hline 10 seconds & $\begin{array}{l}\text { Maximum Utilization } \\
\text { Time }\end{array}$ \\
\hline Application Type & Event Driven \\
\hline
\end{tabular}

The simulation parameters used in TTACCA algorithm is shown in Table 3. The sensor nodes are first deployed randomly in square area with dimensions $500 \mathrm{~m} * 500 \mathrm{~m}$. The sink node is placed in the centre of the area. The WSN is modelled as bowl structure. The transmission range for all nodes is fixed for $212 \mathrm{~m}$. The propagation model used is TwoRayGround. The TTACCA algorithm is simulated under two scenarios: with high load in the network and with low load in the network

\subsection{Performance Analysis}

In this section, we evaluate the performance of TTACCA algorithm using simulation experiments conducted on the NS2 [11] platform. NS2 uses OTcl and C++ codes to implement the given scenario. For a comprehensive performance evaluation, several QoS quantitative metrics considered are defined below

(i) Energy Consumption: The average consumed energy per packet received by the sink reflects the energy efficiency of the protocols. It is ratio of the total energy consumption to the number of packets received by the sink successfully. The lower the energy consumed per packet, the higher the energy efficiency. 


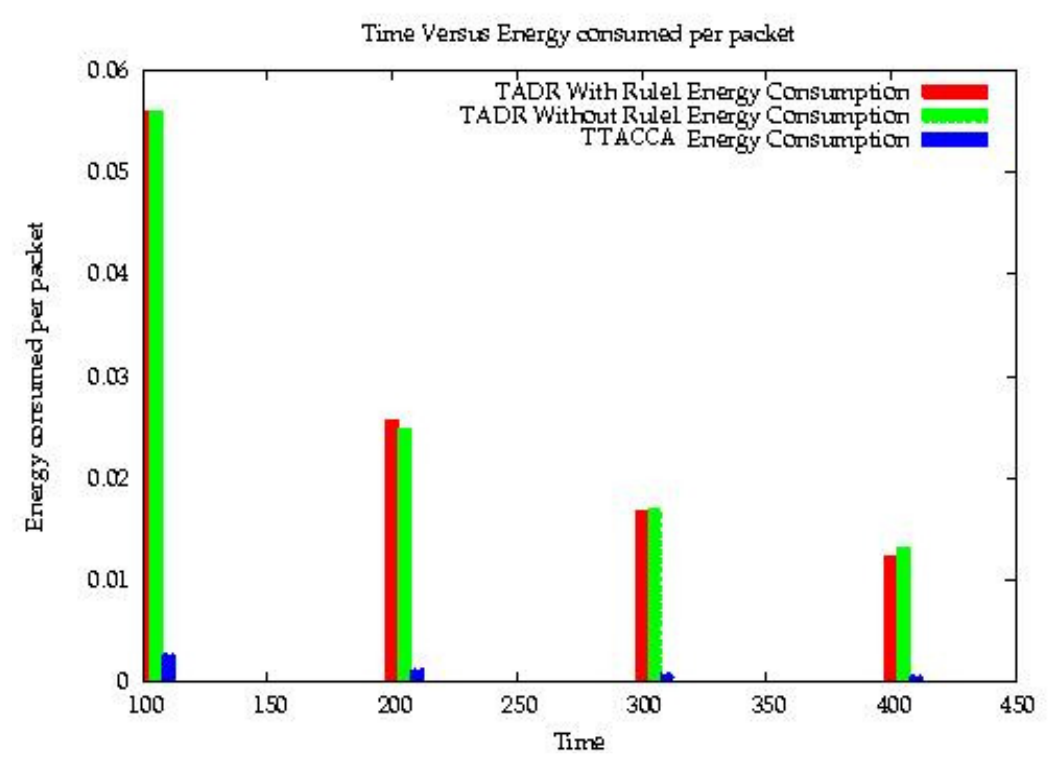

Figure 2: Time versus Throughput

Figure 2 depicts the energy consumption of the TTACCA algorithm. The energy consumed per packet is effectively reduced by $30 \%$ compared to the TADR algorithm. TTACCA algorithm selects the forwarding node by considering distance to sink and forwarder node set information in the one hop neighbourhood or two hop neighbours. Considering distance to sink and forwarder node set information in the one hop neighbourhood or two hop neighbours. The queue length field in the one hop or two hop neighbour set solves the traffic aware problem. The depth filed gives the shortest path from parent node to the destination node. The number of hops travelled by the packet or message to reach the destination is comparatively less. Hence the energy consumed for each packet to reach the destination or sink node is less.

(ii) Throughput: It is the ratio of number of packets received by the sink to the number of packets sent by the source node.

Figure 3 shows the comparison of time versus throughput. TADR routes the packets around the under loaded or idle nodes around the hotspot. Whenever a hotspot occurs in the network this results in packet dropping at the hotspot or near the sink node. Due to this in TADR the overall throughput is comparatively low. TTACCA scatters the packets to under loaded or idle nodes around the hotspot through two hop routing technique or one hop routing technique. Excessive packets in and around the hotspots when congestion occurs in the network are spread evenly through alternate paths with less load. Hence throughput of TTACCA algorithm is improved by $20 \%$ compared with the TADR algorithm. Hence the overall throughput is high compared to TADR 


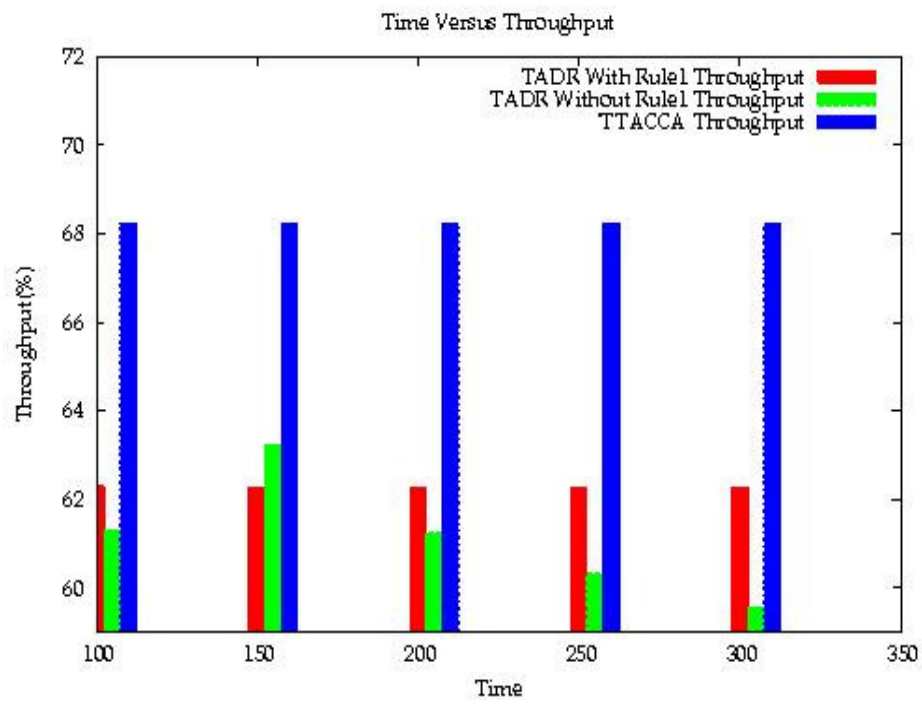

Figure 3: Time versus Throughput

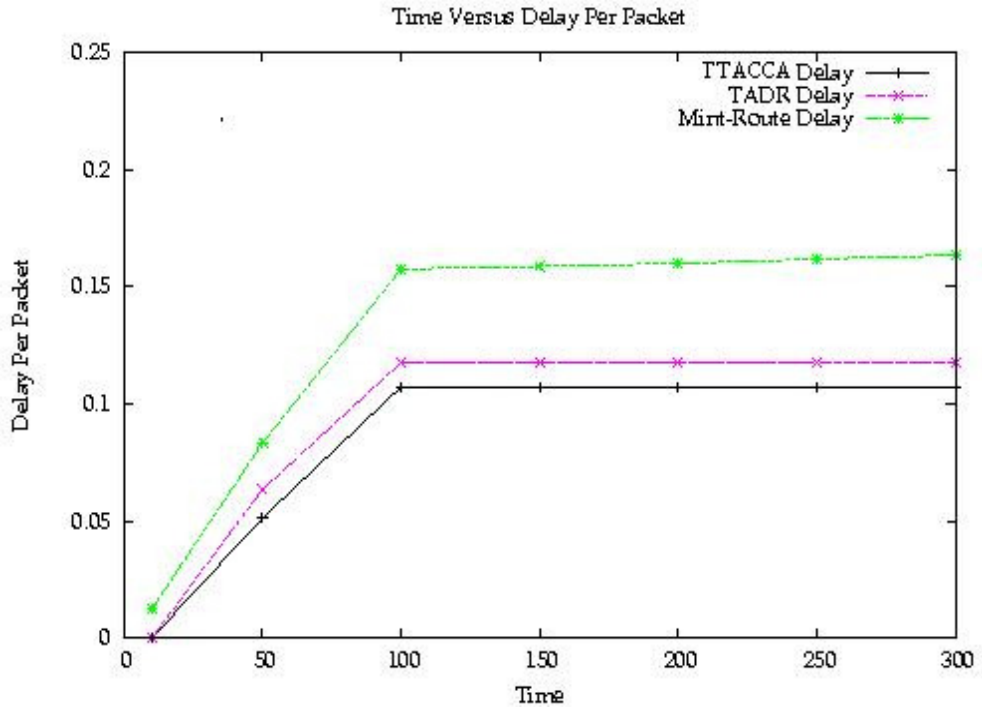

Figure 4: Time versus Delay per Packet

(iii) Delay: The time taken between the source node sending the packet and the destination node receiving the packet is End-to-End Delay.

Figure 4 shows the comparison of delay per packets versus time.Initially no packet transmission takes place due to this the delay is less. As simulation time increases from 0 to 50 seconds, number of packet transmission and transmission overhead increases due to this there is a gradual increase in delay and finally reaches to saturation. In TADR packet transmission is hop by hop. At each hop packet selects its next hop from the one hop neighbour set. Hence dropping of packets is more. Whereas in TTACCA algorithm follows multihop transmission hence packet dropping is low, implies that in TTACCA delay is comparatively low due to the depth potential field, which selects the routing path based on shortest distance from source to the sink node. 


\section{CONCLUSIONS}

The congestion control in WSN is different from that in tradition networks, such as Wireless LAN and adhoc networks. The pure traffic control is able to alleviate congestion, but hard to satisfy the fidelity required by applications. In this paper, TTACCA algorithms follows the philosophy of dynamic capacity planning to deal with the congestion problem in WSN. Through TTACCA algorithm QoS performance metrics delay is reduced by $10 \%$, improves energy efficiency by $30 \%$ and throughput of TTACCA is high compared with TADR. The TACCA algorithm thus mitigates congestion in the network efficiently compared to TADR congestion control algorithm.

\section{REFERENCES}

[1] Mansoor-uz-Zafar Dawood, Biztek, Pakistan,Noor Zaman, Abdul Raouf Khan, Mohammad Salih,"Designing of Energy Aware Quality of Service (QoS) Based Routing Protocol for Efficiency Improvement in Wireless Sensor Network”, Journal of Information and Communication Technology, Vol. 4, No. 1, 2010.

[2] Fengyuan Ren and Tao He, "Traffic-Aware Dynamic Routing to Alleviate Congestion in Wireless Sensor Networks", IEEE Transactions On Parallel and Distributed Systems, Vol. 22, No. 9, September 2011.

[3] Muhammad Monowar, Obaidur Rahman, Al-Sakib Khan Pathan, and Choong Seon Hong, "Prioritized Heterogeneous Traffic-Oriented Congestion Control Protocol for WSNs" , The International Arab Journal of Information Technology, Vol. 9, No. 1, January 2012.

[4] Muhammad Omer Farooq, Thomas Kunz, and Marc St-Hilaire, "Differentiated Services based Congestion Control Algorithm for Wireless Multimedia Sensor Networks", IEEE, 2011.

[5] Sohail Jabbar, Awais Ahmad, Ataul Aziz Ikram, Murad Khan, "TSEEC - TS/TDMA based Energy Efficient Congestion Control in Mobile Wireless Sensor Network", Proceedings of the World Congress on Engineering and Computer Science, October 19-21, 2011, San Francisco, USA 2011.

[6] Charalambos Sergiou, Vasos Vassiliou and Aristodemos Paphitis, "Hierarchical Tree Alternative Path (HTAP) Algorithm for Congestion Control in Wireless Sensor Networks", University of Cyprus, February 3, 2012.

[7] G Srinivasan and S Murugappan, "Rapid Congestion Control Technique for Wireless Sensor Network for Energy Efficiency and Reliability", International Conference on Computing and Control Engineering, 12 and 13 April, 2012.

[8] Hsu-Jung Liu, Mei-Wen Huang, Wen-Shyong Hsieh, Chenhuan Jack Jan,"Priority-based Hybrid Protocol in Wireless Sensor Networks", 11th IEEE International Conference on High performance Computing and Communications, 2009.

[9] Gajendra Sanjay Vyas, Vivek S Deshpande, "Effect of Multiple Sinks on the Performance of Wireless Sensor Networks", International Journal of Emerging Science and Engineering, Vol. 1, No. 5, March 2013.

[10] Kiran Babu T S, Manoj Challa, Sundeep Kumar K, M Jitendranath, " Congestion Control using Adaptive Buffer Flow Managements in WSN", International Journal of Scientific Engineering Research, Vol. 3, No.8, August 2012.

[11] http://www.isi.edu/nsmam/ns 


\section{AUTHORS}

Prabha $\mathbf{R}$ is currently working as an Associate Professor in the Department of Information Science and Engineering, Dr. Ambedkar Institute of Technology, Bangalore, India. She obtained her Bachelor of Engineering degree in Computer Science and Engineering branch. M.E in Computer Science and Engineering from Computer Science Department, UVCE, Bangalore University in the year 2003. She has 22 years of teaching experience. Currently she is pursuing Ph. D in the Department of Computer Science and Engineering, University Visvesvaraya College of Engineering, Bangalore University, Bangalore. Her research interest is in the area of Wireless Sensor Networks.

Prashanth Kumar Gouda received his B.E in Electronics and Communication Engineering from Dr. Ambedkar Institute of Technology Bangalore from Visvesvaraya Technological University in the year of 2010. M.E in Computer Networks from Computer Science Department, UVCE, Bangalore University in the year2013. His research focus includes QoS and Routing in WSN and Cloud Networking and Communication. He worked as Business Intelligence Developer at GrayMatter Software Service Private limited Bangalore.

Dr. S H Manjula is currently working as an Associate Professor in the Department of Computer Science and Engineering, University Visvesvaraya College of Engineering Bangalore University, Bangalore, India. She obtained her Bachelor of Engineering degree in Computer Science and Engineering branch, Masters of Engineering and $\mathrm{Ph}$ D. in Computer Science and Engineering. She has published a book on Wireless Sensor Networks. She has published more than 30 papers in refereed international journals and conferences. Her research interests are in the field of Wireless Sensor Networks, Semantic web and Data Mining.

Dr. Venugopal $\mathbf{K} \mathbf{R}$ is currently Special Officer, DVG Bangalore University and Principal, University Visveswaraya College of Engineering, Bangalore University Bangalore. He obtained his Bachelor of Engineering from University Visvesvaraya College of Engineering. He received his Master's degree in Computer Science and Automation from Indian Institute of Science Bangalore. He was awarded $\mathrm{Ph}$. D in Economics from Bangalore University and Ph.D in Computer Science from Indian Institute of Technology, Madras. He has a distinguished academic career and has degrees in Electronics, Economics, Law, Business Finance, Public Relations, Communications, Industrial Relations, Computer Science and Journalism. He has authored and edited 39 books on Computer Science and Economics, which include Petrodollar and the World Economy, C Aptitude, Mastering C, Microprocessor Programming, Mastering C++ and Digital Circuits and Systems. During his three decades of service at UVCE he has over 400 research papers to his credit. He was a Post-Doctoral Research Scholar at University of Southern California, USA. His research interests include Computer Networks, Wireless Sensor Networks, Parallel and Distributed Systems, Digital Signal Processing and Data Mining.

Dr L M Patnaik is honorary professor in Department of Computer Science and Auto- mation, Indian institute of Science, Bangalore. During the past 35 years of his service at the Institute he has over 700 research publications in refereed International Conference Proceedings. He is a Fellow of all the four leading Science and Engineering Academies in India. Fellow of the IEEE and the Academy of Science for the developing world. He has received twenty national and international awards. Notable among them is the IEEE Technical Achievement Award for his significant contributions to High

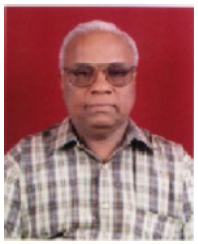
performance Computing and Soft Computing. He is an Ex-Vice Chancellor Defence institute of Advanced Technology, Pune India. His area of research interest has been Parallel and Distributed Computing, Mobile Computing, CAD for VLSI circuits, Soft computing and Computational Neuroscience. 